\title{
NOTES ON TANGENT SPHERE BUNDLES OF CONSTANT RADII
}

\author{
JeongHyeong Park and Kouei Sekigawa
}

\begin{abstract}
We show that the Riemannian geometry of a tangent sphere bundle of a Riemannian manifold $(M, g)$ of constant radius $r$ reduces essentially to the one of unit tangent sphere bundle of a Riemannian manifold equipped with the respective induced Sasaki metrics. Further, we provide some applications of this theorem on the $\eta$-Einstein tangent sphere bundles and certain related topics to the tangent sphere bundles.
\end{abstract}

\section{Introduction}

In the geometry of tangent bundles $T M$ of Riemannian manifolds $M=$ $(M, g)$, the Sasaki (lifted) metric is one of the most natural metrics (denoted it by $\tilde{g}$ on $T M)$ and the Riemannian geometry on $(T M, \tilde{g})$ has been studied by many authors $[1,3,10,11]$. It is also well-known that the Sasaki metric $\tilde{g}$ is compatible with the almost complex structure defined by taking account of the Levi-Civita connection with respect to the metric $g$ and further, $(J, \tilde{g})$ gives rise to an almost Kähler structure on $T M$. Besides the Sasaki metric $\tilde{g}$, there is another well-known Riemannian metric on $T M$ (denoted by $\hat{g}$ ) defined by Cheeger and Gromoll [5]. In the sequel, we shall call it the Cheeger-Gromoll metric on $T M$. The explicit expression of the metric $\hat{g}$ was given by Musso and Tricerri [10]. The tangent sphere bundle $T_{r}(M, g)$ of $(M, g)$ of constant radius $r$ is regarded as a hypersurface of $(T M, \tilde{g})$ and in particular, $T_{1}(M, g)$ is called the unit tangent sphere bundle of $(M, g)$. It is interesting and useful to study the relation between the geometric properties of $(M, g)$ and $T_{r}(M, g)$. We denote the induced Sasaki metric on $T_{r}(M, g)$ by $g_{r}^{\prime}, T_{1}(M, g)$ by $g_{1}^{\prime}$ and the rescaling metric $\left(4 r^{2}\right)^{-1} g_{r}{ }^{\prime}$ by $\overline{g_{r}}$. By making use of the almost Kähler structure $(J, \tilde{g})$, we can define the so-called standard contact metric structure $\left(\overline{g_{r}}, \phi, \xi, \eta\right)$. In our previous paper [4], we discussed the problem, "when is $T_{1}(M, g)$ equipped with the standard contact metric structure $\eta$-Einstein?", and also raised the

Received April 17, 2008.

2000 Mathematics Subject Classification. 53C25, 53D10, 53B20.

Key words and phrases. tangent sphere bundle, contact metric structure, Sasaki metric, $\eta$-Einstein manifold.

This work was supported by the Korea Science and Engineering Foundation(KOSEF) grant funded by the Korea Government(MEST) (R01-2008-000-20370-0). 
similar problem for $\left(T_{r}(M, g), \overline{g_{r}}, \phi, \xi, \eta\right)$ [7]. Taking account of the arguments in $[2,6,8,9,11]$, it is natural to consider the relations among the tangent sphere bundles of a Riemannian manifold of various constant radii equipped with the induced Sasaki metrics and also the induced Cheeger-Gromoll metrics. We refer to [1] for a related discussion of the natural metrics on the unit sphere bundles. In [1], the metric on unit sphere bundle depends essentially on the Sasaki metric and also on the vertical lift of the base metric. Contrary to this, the metrics in this paper are defined on the common manifold $T_{r}(M, g)=T_{1}\left(M, r^{-2} g\right)$ and depend only on the Sasaki metrics with respect to $g$ and $r^{-2} g$ on the base manifold $M$. This is the primary difference from Abassi-Kowalski's work [1]. The main theorem of this paper is the following:

Theorem 1. Let $M=(M, g)$ be a Riemannian manifold. Then $\left(T_{r}(M, g), g_{r}^{\prime}\right)$ is homothetic to $\left(T_{1}\left(M, r^{-2} g\right),\left(r^{-2} g\right)_{1}^{\prime}\right)$ with the constant scaling factor $r^{2}$ under the identity map.

In Section 3, we shall prove Theorem 1, and give some applications. Finally, in Section 4, we shall give remarks related to the theorems given in Section 3.

The authors would like to express their thanks to Professors Kowalski, Abassi and Sekizawa for their helpful comments and assistance with this paper.

\section{Tangent sphere bundles of constant radii}

Let $M=(M, g)$ be an $n$-dimensional Riemannian manifold and $\nabla$ the Levi-Civita connection of $g$. Its Riemannian curvature tensor $R$ is defined by $R(X, Y) Z=\left[\nabla_{X}, \nabla_{Y}\right] Z-\nabla_{[X, Y]} Z$ for all vector fields $X, Y$ and $Z$ on $M$. The tangent bundle of $M$ is denoted by $T M$ and consists of pairs $(p, u)$, where $p$ is a point in $M$ and $u$ is a tangent vector to $M$ at $p$. We denote by $\pi$ the natural projection $\pi: T M \longrightarrow M, \pi(p, u)=p$. For a vector field $X$ on $M$, its vertical lift $X^{v}$ on $T M$ is the vertical vector field defined by $X^{v} \omega=\omega(X) \circ \pi$, where $\omega$ is any 1 -form on $M$. For the Levi-Civita connection $\nabla$ on $M$, the horizontal lift $X^{h}$ of $X$ is defined by $X^{h} \omega=\nabla_{X} \omega$. The tangent bundle $T M$ can be endowed in a natural way with a Riemannian metric $\tilde{g}$, the so-called Sasaki (lifted) metric, depending only on the Riemannian metric $g$ on $M$. It is determined by

$$
\tilde{g}\left(X^{h}, Y^{h}\right)=\tilde{g}\left(X^{v}, Y^{v}\right)=g(X, Y) \circ \pi, \quad \tilde{g}\left(X^{v}, Y^{h}\right)=0
$$

for all tangent vector fields $X, Y$ on $M$. Also, $T M$ admits an almost complex structure $J$ defined by $J X^{h}=X^{v}$ and $J X^{v}=-X^{h}$. Then it is well-known that $(J, \tilde{g})$ is an almost Kähler structure on $T M$. The tangent sphere bundle $\bar{\pi}: T_{r}(M, g) \longrightarrow M$ of constant radius $r$ is a hypersurface of $T M$ given by $g_{p}(u, u)=r^{2}$ for all $p \in M$. Note that $\bar{\pi}=\pi \circ i$, where $i$ is the inclusion map. A unit normal vector field $N$ to $T_{r}(M, g)$ is given by $N=\frac{1}{r} u^{v}$ for $(p, u) \in T_{r}(M, g)$. The horizontal lift of any vector of $M$ is necessarily tangent to $T_{r}(M, g)$, but the vertical lift is not tangent to $T_{r}(M, g)$ in general. So, we 
define the tangential lift of a vector $X$ to $(p, u) \in T_{r}(M, g)$ by

$$
X_{(p, u)}^{t}=X^{v}-\frac{1}{r^{2}} g(X, u) u^{v} .
$$

Clearly, the tangent space $T_{(p, u)}\left(T_{r}(M, g)\right)$ is spanned by the vectors of the forms $X^{h}$ and $X^{v}$ for $X \in T_{p}(M)(p \in M)$. With the induced Sasaki metric $g_{r}^{\prime}$ on $T_{r}(M, g)$, taking account of $(2.2)$, we have

$$
\begin{aligned}
g_{r}^{\prime}\left(X^{t}, Y^{t}\right) & =g(X, Y)-\frac{1}{r^{2}} g(X, u) g(Y, u), \\
g_{r}^{\prime}\left(X^{t}, Y^{h}\right) & =0, \\
g_{r}^{\prime}\left(X^{h}, Y^{h}\right) & =g(X, Y)
\end{aligned}
$$

for all vector fields $X, Y$ on $M$. We denote by $\nabla^{\prime}$ the Levi-Civita connection of $g_{r}^{\prime}$ on $T_{r}(M, g)$. Then $\nabla^{\prime}$ is given by

$$
\begin{aligned}
\nabla_{X^{t}}^{\prime} Y^{t} & =-\frac{1}{r^{2}} g(Y, u) X^{t}, \\
\nabla_{X^{t}}^{\prime} Y^{h} & =\frac{1}{2}(R(u, X) Y)^{h}, \\
\nabla_{X^{h}}^{\prime} Y^{t} & =\left(\nabla_{X} Y\right)^{t}+\frac{1}{2}(R(u, Y) X)^{h}, \\
\nabla_{X^{h}}^{\prime} Y^{h} & =\left(\nabla_{X} Y\right)^{h}-\frac{1}{2}(R(X, Y) u)^{t}
\end{aligned}
$$

for all vector fields $X, Y$ on $M$. Further, the curvature tensor $R^{\prime}$ of $\left(T_{r}(M, g)\right.$, $\left.g_{r}^{\prime}\right)$ is given by

$$
\begin{aligned}
R^{\prime}\left(X^{t}, Y^{t}\right) Z^{t}= & -\frac{1}{r^{2}}\left(g(X, Z)-\frac{1}{r^{2}} g(X, u) g(Z, u)\right) Y^{t} \\
& +\frac{1}{r^{2}}\left(g(Y, Z)-\frac{1}{r^{2}} g(Y, u) g(Z, u)\right) X^{t} \\
R^{\prime}\left(X^{t}, Y^{t}\right) Z^{h}= & \left\{R\left(X-\frac{1}{r^{2}} g(X, u) u, Y-\frac{1}{r^{2}} g(Y, u) u\right) Z\right\}^{h} \\
& +\frac{1}{4}\{[R(u, X), R(u, Y)] Z\}^{h}, \\
R^{\prime}\left(X^{h}, Y^{t}\right) Z^{t}= & -\frac{1}{2}\left\{R\left(Y-\frac{1}{r^{2}} g(Y, u) u, Z-\frac{1}{r^{2}} g(Z, u) u\right) X\right\}^{h} \\
& -\frac{1}{4}\{R(u, Y) R(u, Z) X\}^{h}, \\
R^{\prime}\left(X^{h}, Y^{t}\right) Z^{h}= & \frac{1}{2}\left\{R(X, Z)\left(Y-\frac{1}{r^{2}} g(Y, u) u\right)\right\}^{t}-\frac{1}{4}\{R(X, R(u, Y) Z) u\}^{t} \\
& +\frac{1}{2}\{(\nabla \times R)(u, Y) Z\}^{h},
\end{aligned}
$$




$$
\begin{aligned}
R^{\prime}\left(X^{h}, Y^{h}\right) Z^{t}= & \left\{R(X, Y)\left(Z-\frac{1}{r^{2}} g(Z, u) u\right)\right\}^{t} \\
& +\frac{1}{4}\{R(Y, R(u, Z) X) u-R(X, R(u, Z) Y) u\}^{t} \\
& +\frac{1}{2}\left\{\left(\nabla_{X} R\right)(u, Z) Y-\left(\nabla_{Y} R\right)(u, Z) X\right\}^{h}, \\
R^{\prime}\left(X^{h}, Y^{h}\right) Z^{h}= & (R(X, Y) Z)^{h}+\frac{1}{2}\{R(u, R(X, Y) u) Z\}^{h} \\
& -\frac{1}{4}\{R(u, R(Y, Z) u) X-R(u, R(X, Z) u) Y\}^{h} \\
& +\frac{1}{2}\left\{\left(\nabla_{Z} R\right)(X, Y) u\right\}^{t}
\end{aligned}
$$

for all vector fields $X, Y$ and $Z$ on $M$.

Further, we denote by $\rho^{\prime}$ and $\tau^{\prime}$ the Ricci tensor and the scalar curvature of $\left(T_{r}(M, g), g_{r}^{\prime}\right)$, respectively. To calculate the Ricci tensor $\rho^{\prime}$ at the point $(p, u) \in T_{r}(M, g)$, we choose an orthonormal basis $\left\{e_{1}, \ldots, e_{n}=\frac{u}{r}\right\}$. Then $\left\{e_{1}^{t}, \ldots, e_{n-1}^{t}, e_{1}^{h}, \ldots, e_{n}^{h}\right\}$ is an orthonormal basis for $T_{(p, u)}\left(T_{r}(M, g)\right)$, and from (2.5), $\rho^{\prime}$ is given by

$$
\begin{aligned}
\rho^{\prime}\left(X^{t}, Y^{t}\right)= & \frac{n-2}{r^{2}}\left(g(X, Y)-\frac{1}{r^{2}} g(X, u) g(Y, u)\right) \\
& +\frac{1}{4} \sum_{i=1}^{n} g\left(R(u, X) e_{i}, R(u, Y) e_{i}\right), \\
\rho^{\prime}\left(X^{t}, Y^{h}\right)= & \frac{1}{2}\left(\left(\nabla_{u} \rho\right)(X, Y)-\left(\nabla_{X} \rho\right)(u, Y)\right), \\
\rho^{\prime}\left(X^{h}, Y^{h}\right)= & \rho(X, Y)-\frac{1}{2} \sum_{i=1}^{n} g\left(R\left(u, e_{i}\right) X, R\left(u, e_{i}\right) Y\right)
\end{aligned}
$$

for all vector fields $X, Y$ on $M$. From (2.6), we have further

$$
\tau^{\prime}=\tau+\frac{(n-1)(n-2)}{r^{2}}-\frac{1}{4} \sum_{i, j}^{n} g\left(R\left(u, e_{i}\right) e_{j}, R\left(u, e_{i}\right) e_{j}\right),
$$

where $\tau$ is the scalar curvature of $M$. In the rest of this section, we shall review the definition of the standard contact metric structure on $T_{r}(M, g)$. First, using the almost complex structure $J$ on $T M$, we define a unit vector field $\xi^{\prime}$ (with respect to the metric $\left.g_{r}^{\prime}\right)$, a 1 -form $\eta^{\prime}$ and a $(1,1)$-tensor field $\phi^{\prime}$ on $T_{r}(M, g)$ by

$$
\xi^{\prime}=-J N, \quad \phi^{\prime}=J-\eta^{\prime} \otimes N .
$$

Since $g^{\prime}\left(\bar{X}, \phi^{\prime} \bar{Y}\right)=2 r d \eta^{\prime}(\bar{X}, \bar{Y}),\left(g^{\prime}, \phi^{\prime}, \eta^{\prime}, \xi^{\prime}\right)$ is not a contact metric structure on $T_{r}(M, g)$. By rescaling as

$$
\xi=2 r \xi^{\prime}, \quad \eta=\frac{1}{2 r} \eta^{\prime}, \quad \phi=\phi^{\prime}, \quad \overline{g_{r}}=\frac{1}{4 r^{2}} g^{\prime}
$$


we get the standard contact metric structure $\left(\overline{g_{r}}, \phi, \xi, \eta\right)$. These tensors are given by

$$
\begin{aligned}
& \xi=2 u^{h}, \\
& \phi X^{t}=-X^{h}+\frac{1}{2 r^{2}} g(X, u) \xi, \quad \phi X^{h}=X^{t}, \\
& \eta\left(X^{t}\right)=0, \quad \eta\left(X^{h}\right)=\frac{1}{2 r^{2}} g(X, u), \\
& \bar{g}\left(X^{t}, Y^{t}\right)=\frac{1}{4 r^{2}}\left(g(X, Y)-\frac{1}{r^{2}} g(X, u) g(Y, u)\right), \\
& \bar{g}\left(X^{t}, Y^{h}\right)=0, \\
& \bar{g}\left(X^{h}, Y^{h}\right)=\frac{1}{4 r^{2}} g(X, Y),
\end{aligned}
$$

where $X, Y$ are vector fields on $M$. Comparing (2.4), (2.5), and (2.6) with the formulas (2.3), (2.4), and (2.5) in [7], we see that $\bar{\nabla}=\nabla^{\prime}, \bar{R}=R^{\prime}$ and $\bar{\rho}=\rho^{\prime}$. The scalar curvature $\bar{\tau}$ of the metric $\overline{g_{r}}$ is given by

$$
\bar{\tau}=4 r^{2} \tau+4(n-1)(n-2)-r^{2} \sum_{i, j=1}^{n} g\left(R\left(u, e_{i}\right) e_{j}, R\left(u, e_{i}\right) e_{j}\right),
$$

where $\tau$ is the scalar curvature of $M$.

Since $\rho^{\prime}=\bar{\rho}$ holds, from (2.7) and (2.11), we have

$$
\bar{\tau}=4 r^{2} \tau^{\prime}
$$

\section{Proof of the main theorem and applications}

Let $M=(M, g)$ be an $n$-dimensional Riemannian manifold, and $T_{r}(M, g)$ and $T_{1}\left(M, r^{-2} g\right)$ be the tangent sphere bundle of constant radius $r$ of $(M, g)$ and the unit tangent sphere bundle of $\left(M, r^{-2} g\right)$ equipped respectively with induced Sasaki metrics $g_{r}^{\prime}$ and $\left(r^{-2} g\right)_{1}^{\prime}$. We see easily that the manifolds $T_{r}(M, g)$ and $T_{1}\left(M, r^{-2} g\right)$ can be naturally identified. Next, from (2.2), we may also easily check that the tangential lifts of a vector $X$ of $M$ to $(p, u) \in T_{r} M$ with respect to the metrics $g_{r}^{\prime}$ and $\left(r^{-2} g\right)_{1}^{\prime}$ coincide, and hence, we denote it by using the common letter $X_{(p, u)}^{t}$. The similar fact is also valid for the horizontal lifts since the Levi-Civita connections of the Riemannian metrics $g$ and $r^{-2} g$ coincide. From these observations and (2.3), we have

$$
\begin{aligned}
\left(\frac{1}{r^{2}} g\right)_{1}^{\prime}\left(X^{t}, Y^{t}\right) & =\left(\frac{1}{r^{2}} g\right)(X, Y)-\left(\frac{1}{r^{2}} g\right)(X, u)\left(\frac{1}{r^{2}} g\right)(Y, u) \\
& =\frac{1}{r^{2}} g_{r}^{\prime}\left(X^{t}, Y^{t}\right) \\
\left(\frac{1}{r^{2}} g\right)_{1}^{\prime}\left(X^{t}, Y^{h}\right) & =0=\frac{1}{r^{2}} g_{r}^{\prime}\left(X^{t}, Y^{h}\right)
\end{aligned}
$$




$$
\left(\frac{1}{r^{2}} g\right)_{1}^{\prime}\left(X^{h}, Y^{h}\right)=\frac{1}{r^{2}} g(X, Y)=\frac{1}{r^{2}} g_{r}^{\prime}\left(X^{h}, Y^{h}\right)
$$

hold for all vector fields $X, Y$ on $M$. This completes the proof of Theorem 1 .

(I) Application 1.

Let $M=(M, g)$ be a 2-dimensional Riemannian manifold and $T_{1}(M, g)$ be the unit tangent sphere bundle of $M$ equipped with the induced Sasaki metric $g_{1}^{\prime}$. Let $\left\{e_{1}, e_{2}=u\right\}$ be an orthonormal basis at $p \in M$ and $\left\{e_{i}^{\prime}\right\}$ the basis at $(p, u) \in T_{1}(M, g)$ given by

$$
e_{1}^{\prime}=e_{1}^{t}, e_{2}^{\prime}=e_{1}^{h}, e_{3}^{\prime}=e_{2}^{h} .
$$

Then, from (2.6), we have

$$
\begin{gathered}
\rho^{\prime}\left(e_{1}^{\prime}, e_{1}^{\prime}\right)=\frac{1}{2} \kappa^{2}, \\
\rho^{\prime}\left(e_{2}^{\prime}, e_{2}^{\prime}\right)=\rho^{\prime}\left(e_{3}^{\prime}, e_{3}^{\prime}\right)=\kappa-\frac{1}{2} \kappa^{2}, \\
\rho^{\prime}\left(e_{1}^{\prime}, e_{2}^{\prime}\right)=\frac{1}{2} e_{2} \kappa, \rho^{\prime}\left(e_{1}^{\prime}, e_{3}^{\prime}\right)=-\frac{1}{2} e_{1} \kappa, \\
\rho^{\prime}\left(e_{2}^{\prime}, e_{3}^{\prime}\right)=0,
\end{gathered}
$$

where $\kappa$ is the Gaussian curvature of $M$. From (3.3), we have further

$$
\tau^{\prime}=2 \kappa-\frac{\kappa^{2}}{2}=\frac{\kappa}{2}(4-\kappa)
$$

Thus, from (3.4) and Theorem 1, we have the following:

Theorem 2. Let $M=(M, g)$ be a 2-dimensional Riemannian manifold of constant Gaussian curvature $c$. Then, the scalar curvature $\tau^{\prime}$ of the unit tangent sphere bundle $T_{1}(M, g)=\left(T_{1}(M, g), g_{1}^{\prime}\right)$ is constant and satisfies the following relations:

(1) If $c>0$, then $\tau^{\prime}>0$ for $c<4, \tau^{\prime}=0$ for $c=4, \tau^{\prime}<0$ for $c>4$.

(2) If $c \leq 0$, then $\tau^{\prime} \leq 0$.

Thus, from Theorems 1 and 2, we have the following:

Corollary 3 ([10, Corollary 3.4]). Let $M=(M, g)$ be a 2-dimensional Riemannian manifold of constant Gaussian curvature $c$. Then, the scalar curvature $\tau^{\prime}$ of the tangent sphere bundle $\left(T_{r}(M, g), g_{r}^{\prime}\right)$ is constant and satisfies the following relations:

(1) If $c>0$, then $\tau^{\prime}>0$ for $r<\frac{2}{\sqrt{c}}, \tau^{\prime}=0$ for $r=\frac{2}{\sqrt{c}}, \tau^{\prime}<0$ for $r>\frac{2}{\sqrt{c}}$.

(2) If $c \leq 0$, then $\tau^{\prime} \leq 0$ for all $r$.

Kowalski and Sekizawa [9] extended Corollary 3 for arbitrary dimension.

(II) Application 2.

A contact metric manifold $\bar{M}=(\bar{M}, \bar{g}, \phi, \xi, \eta)$ is called an $\eta$-Einstein manifold if the Ricci tensor $\rho$ is of the form $\bar{\rho}=\alpha \bar{g}+\beta \eta \otimes \eta$ with $\alpha$ and $\beta$ being 
smooth functions. Based upon the theorems that $\alpha$ and $\beta$ are constant on the $\eta$-Einstein tangent sphere bundles with constant radii $[4,7]$ and Theorem 1 , we may have the following:

Theorem 4. $\left(T_{r}(M, g), \bar{g}_{r}, \phi, \xi, \eta\right)$ is isomorphic with $\left(T_{1}\left(M, r^{-2} g\right), \overline{\left(r^{-2} g\right)}{ }_{1}\right.$, $\phi, \xi, \eta)$ as a contact metric manifold under the identity map, and hence, in particular, $\left(T_{r}(M, g), \bar{g}_{r}, \phi, \xi, \eta\right)$ is $\eta$-Einstein if and only if $\left(T_{1}\left(M, r^{-2} g\right)\right.$, \left.${\overline{\left(r^{-2} g\right)}}_{1}, \phi, \xi, \eta\right)$ is $\eta$-Einstein.

In [4], we prove that the unit tangent sphere bundle $\left(T_{1} M, \bar{g}_{1}, \phi, \xi, \eta\right)$ of a 2-dimensional Riemannian manifold $M=(M, g)$ is $\eta$-Einstein if and only if $M$ is of constant Gaussian curvature $\kappa=0$ or 1 . Thus, taking account of Theorem 4 , we see that $\left(T_{r} M, \bar{g}_{r}, \phi, \xi, \eta\right)$ is $\eta$-Einstein if and only if $M$ is of constant Gaussian curvature $\kappa=0$ or $\frac{1}{r^{2}}[7]$.

(III) Application 3.

The Cheeger-Gromoll metric (denoted it by $\hat{g}$ ) on the tangent bundle $T M$ of a Riemannian manifold $(M, g)$ is defined by

$$
\begin{aligned}
& \hat{g}\left(X^{v}, Y^{v}\right)=\frac{1}{1+r^{2}}(g(X, Y)+g(X, u) g(Y, u)), \\
& \hat{g}\left(X^{v}, Y^{h}\right)=0 \\
& \hat{g}\left(X^{h}, Y^{h}\right)=g(X, Y)
\end{aligned}
$$

at each point $(p, u) \in T M$ for all tangent vectors $X, Y$ at $p \in M$. Here $r=|u|$ $([10,11])$.

Further, we denote by $\hat{g}_{r}^{\prime}$ the induced Cheeger-Gromoll metric on $T_{r}(M, g)$. Then, from (2.2) and (3.5), we may easily check that the metric $\hat{g}_{r}^{\prime}$ is given explicitly by

$$
\begin{aligned}
\hat{g}_{r}^{\prime}\left(X^{t}, Y^{t}\right) & =\frac{1}{1+r^{2}}\left(g(X, Y)-\frac{1}{r^{2}} g(X, u) g(Y, u)\right) \\
\hat{g}_{r}^{\prime}\left(X^{t}, Y^{h}\right) & =0 \\
\hat{g}_{r}^{\prime}\left(X^{h}, Y^{h}\right) & =g(X, Y)
\end{aligned}
$$

at each point $(p, u) \in T_{r}(M, g)$ for all tangent vectors $X, Y$ on $M$. Now, we define the diffeomorphism $f: T_{r}(M, g) \rightarrow T_{\frac{r}{\sqrt{1+r^{2}}}}(M, g)$ defined by

$$
f:(p, u) \longmapsto\left(p, \frac{u}{\sqrt{1+r^{2}}}\right) .
$$

From $(3.5)$, since $\hat{g}_{(p, u)}\left(u^{v}, u^{v}\right)=r^{2}(r=|u|)$ for any $u \in T_{p} M$, we may observe that the tangential lift of a vector $X$ of $M$ to $(p, u) \in T_{r}(M, g)$ with respect to the Cheeger-Gromoll metric $\hat{g}$ takes the same form as (2.2). By the definition 
of the map $f$, we have

$$
\begin{aligned}
& \left(f_{*}\right)_{(p, u)}\left(X^{t}\right)_{(p, u)}=\frac{1}{\sqrt{1+r^{2}}}\left(X^{t}\right)_{\left(p, \frac{u}{\sqrt{1+r^{2}}}\right)}, \\
& \left(f_{*}\right)_{(p, u)}\left(X^{h}\right)_{(p, u)}=\left(X^{h}\right)_{\left(p, \frac{u}{\sqrt{1+r^{2}}}\right)}
\end{aligned}
$$

for all tangent vector $X$ of $M$ and any $(p, u) \in T_{r} M$. Thus, from (3.8), we have

$$
\begin{aligned}
& \left(g_{\frac{r}{\sqrt{1+r^{2}}}}\right)_{\left(p, \frac{u}{\sqrt{1+r^{2}}}\right)}\left(\left(f_{*}\right)_{(p, u)}\left(X^{t}\right)_{(p, u)},\left(f_{*}\right)_{(p, u)}\left(Y^{t}\right)_{(p, u)}\right) \\
= & \frac{1}{1+r^{2}}\left(g(X, Y)-\frac{1}{r^{2}} g(X, u) g(Y, u)\right)=\hat{g}_{(p, u)}^{\prime}\left(X^{t}, Y^{t}\right), \\
& \left(g_{\frac{r}{\sqrt{1+r^{2}}}}\right)^{\prime}\left(p, \frac{u}{\sqrt{1+r^{2}}}\right) \\
= & \frac{1}{\sqrt{1+r^{2}}} \hat{g}_{(p, u)}^{\prime}\left(X^{t}, Y^{h}\right)=0, \\
& \left.\left(g_{\frac{r}{\sqrt{1+r^{2}}}}\right)_{(p, u)}^{\prime}\left(X^{t}\right)_{(p, u)},\left(f_{*}\right)_{(p, u)}\left(Y^{h}\right)_{(p, u)}\right) \\
= & \hat{g}_{(p, u)}^{\prime}\left(X^{h}, Y^{h}\right)=g(X, Y),
\end{aligned}
$$

at any point $(p, u) \in T_{r} M$ for all tangent vectors $X, Y$ of $M$ at $p \in M$.

Thus, from (3.9), we see that $f$ is an isometry from $\left(T_{r}(M, g), \hat{g}_{r}^{\prime}\right)$ onto $\left(T \frac{r}{\sqrt{1+r^{2}}}(M, g), g_{\frac{r}{\sqrt{1+r^{2}}}}^{\prime}\right)$. This fact was already announced by Boeckx [2]. Thus, taking account of Theorem 1, we have the following:

Theorem 5. Let $(M, g)$ be a Riemannian manifold. Then the tangent sphere bundle $\left(T_{r}(M, g), \hat{g}^{\prime}\right)$ of constant radius $r$ is homothetic to the unit tangent sphere bundle $\left(T_{1}\left(M, \frac{1+r^{2}}{r^{2}} g\right),\left(\frac{1+r^{2}}{r^{2}} g\right)^{\prime}\right)$ with the constant scaling factor $\frac{r^{2}}{1+r^{2}}$ through the diffeomorphism $f$ given by (3.7), where $\hat{g}^{\prime}$ and $\left(\frac{1+r^{2}}{r^{2}} g\right)^{\prime}$ are the induced Cheeger-Gromoll metrics on $T_{r}(M, g)$ and the induced Sasaki metric on $T_{1}\left(M, \frac{1+r^{2}}{r^{2}} g\right)$, respectively.

\section{Remarks}

Let $(M, g)$ be an $n$-dimensional Riemannian manifold and $r>0$ be a smooth function on $M$. We consider the tangent sphere bundle $\left(T_{r}(M, g), \overline{g_{r}}\right)$ of variable radius $r$ and the unit tangent sphere bundle $\left(T_{1}\left(M, r^{-2} g\right),\left(\overline{r^{-2} g}\right)_{1}\right)$ over $M$. Here, we adopt the similar notational conventions as in the case of constant radii. We recall that the manifolds $T_{r}(M, g)$ and $T_{1}\left(M, r^{-2} g\right)$ can be naturally identified. We set $g^{*}=r^{-2} g$, and denote the tangential lift and the horizontal 
lift of a tangent vector $X$ at a point $p \in M$ to the point $u \in T_{1}\left(M, g^{*}\right)$ by $X^{t *}$ and $X^{h *}$ with respect to $\left(T\left(M, g^{*}\right), \tilde{g}^{*}\right)$ respectively. Then, we have

$$
\begin{aligned}
X^{t *} & =X^{t}, \\
X^{h *} & =X^{h}+\left(\frac{X r}{r}\right) u^{v}+\left(\frac{u r}{r}\right) X^{v}-g(X, u)\left(\frac{\nabla r}{r}\right)^{v}
\end{aligned}
$$

at any point $(p, u) \in T_{1}\left(M, g^{*}\right)$, where $X^{h}$ denotes the horizontal lift of $X$ with respect to $(T(M, g), \tilde{g})$, and $\nabla r$ denotes the gradient vector of $r$. From (4.1), (2.2), (2.3), and (2.9), by direct calculations, we have the following:

$$
\begin{aligned}
\left(\overline{g^{*}}\right)_{1}\left(X^{t}, Y^{t}\right)= & \left(\overline{g^{*}}\right)_{1}\left(X^{t *}, Y^{t *}\right)=\frac{1}{4 r^{2}}\left(g(X, Y)-\frac{1}{r^{2}} g(X, u) g(Y, u)\right) \\
= & \bar{g}_{r}\left(X^{t}, Y^{t}\right), \\
\left(\overline{g^{*}}\right)_{1}\left(X^{t *}, Y^{h *}\right)= & 0 \\
\overline{g_{r}}\left(X^{t *}, Y^{h *}\right)= & \frac{1}{4 r^{2}} g^{\prime}\left(X^{v}-\frac{1}{r^{2}} g(X, u) u^{v}, Y^{h}+\frac{Y r}{r} u^{v}\right. \\
& \left.+\frac{u r}{r} Y^{v}-\frac{g(Y, u)}{r}(\nabla r)^{v}\right) \\
= & \frac{1}{4 r^{2}}\left(\frac{u r}{r} g(X, Y)-\frac{g(Y, u)}{r} X r\right), \\
\left(\overline{g^{*}}\right)_{1}\left(X^{h *}, Y^{h *}\right)= & \frac{1}{4 r^{2}} g(X, Y), \\
\overline{g_{r}}\left(X^{h *}, Y^{h *}\right)= & \frac{1}{4 r^{2}} g(X, Y)+\frac{1}{4 r^{2}}\left\{(X r)(Y r)+\frac{(u r)^{2}}{r^{2}} g(X, Y)\right. \\
& \left.+\frac{g(X, u) g(Y, u)}{r^{2}}|\nabla r|^{2}-\frac{u r}{r^{2}} g(Y, u) X r-\frac{u r}{r^{2}} g(X, u) Y r\right\}
\end{aligned}
$$

for any tangent vectors $X, Y$ and $u \in T_{r}(M, g)$.

From (4.2), we immediately have the following:

Theorem 6. Let $r$ be a positive valued smooth function on $M$. Then, $\left(T_{r}(M, g)\right.$, $\left.\overline{g_{r}}\right)$ is isometric to $\left(T_{1}\left(M, g^{*}\right),\left(\overline{g^{*}}\right)_{1}\right)$ under the identity map if and only if $r$ is a constant valued function on $M$, where $g^{*}=r^{-2} g$.

We may define an almost contact metric structure $\left(\bar{g}_{r}, \phi, \xi, \eta\right)$ on $T_{r}(M, g)$ in a similar way as in the case of the constant radius which is called the standard contact metric structure. We denote by $\left(\overline{g^{*}}, \phi^{*}, \xi^{*}, \eta^{*}\right)$ the standard contact metric structure on $T_{1}\left(M, g^{*}\right)$. Let $N$ and $N^{*}$ be the unit vectors to $T_{r}(M, g)\left(=T_{1}\left(M, g^{*}\right)\right)$ with respect to the Riemannian metric $\tilde{g}$ and $\tilde{g}^{*}$ on $T M$, respectively. Then, taking account of (4.1), we see that $N$ and $N^{*}$ are give by

$$
N=\frac{1}{r \sqrt{1+|\nabla r|^{2}}}\left(u^{v}-r(\nabla r)^{h}\right), \quad N^{*}=u^{v} .
$$


We see also that the vectors $X^{t}$ and $X^{h^{\prime}} \equiv X^{h}+\frac{X r}{r} u^{v}$ are tangent to $T_{r}(M, g)$. Thus, from (4.1) and (4.3), by direct computation, we have the following equalities:

$$
\begin{aligned}
\xi= & \frac{2}{\sqrt{1+|\nabla r|^{2}}}\left(u^{h}+r(\nabla r)^{v}\right) \\
\xi^{*}= & 2 u^{h}+\frac{4 u r}{r} u^{v}-2 r(\nabla r)^{v} \\
\phi X^{t}= & -X^{h}+\frac{1}{r^{2}} g(X, u) u^{h} \\
& +\frac{r^{2} X r-g(X, u) u r}{r^{2}\left(1+|\nabla r|^{2}\right)}(\nabla r)^{h}-\frac{r^{2} X r-g(X, u) u r}{r^{3}\left(1+|\nabla r|^{2}\right)} u^{v} \\
\phi^{*} X^{t}= & -X^{h}+\frac{1}{r^{2}} g(X, u) u^{h}-\frac{X r}{r} u^{v}-\frac{u r}{r} X^{v}+\frac{2 u r}{r^{3}} g(X, u) u^{v}, \\
\phi X^{h *}= & X^{v}-\frac{X r}{r} u^{h}-\frac{u r}{r} X^{h}-\frac{2(X r) u r+\left(1-|\nabla r|^{2}\right) g(X, u)}{r^{2}\left(1+|\nabla r|^{2}\right)} u^{v} \\
& +\frac{2(g(X, u)+(X r) u r)}{1+|\nabla r|^{2}}\left(\frac{\nabla r}{r}\right)^{h}, \\
\phi^{*} X^{h *}= & X^{t} .
\end{aligned}
$$

Thus, from (4.4) and Theorem 6, we have immediately the following:

Theorem 7. Let $r$ be a smooth positive function on $M$. Then, the almost contact structure $(\phi, \xi, \eta)$ and the contact structure $\left(\phi^{*}, \xi^{*}, \eta^{*}\right)$ on $T_{r}(M, g)$ coincide if and only if $r$ is constant on $M$. If $r$ is constant on $M$, then the contact metric structures $\left(\overline{g_{r}}, \phi, \xi, \eta\right)$ and $\left(\overline{\left(g^{*}\right)}, \phi^{*}, \xi^{*}, \eta^{*}\right)\left(g^{*}=r^{-2} g\right)$ coincide.

Relating to the Theorems 4, 6, and 7, the following question naturally arises.

Question. Does there exist a non-constant positive valued smooth function $r$ on $M$ such that the almost contact structure $(\phi, \xi, \eta)$ (resp. the almost contact metric structure $\left.\left(\overline{g_{r}}, \phi, \xi, \eta\right)\right)$ is a contact structure (resp. a contact metric structure) on $T_{r}(M, g)$ ?

Further, it is also to worthwhile to discuss the relation between the standard almost contact metric structure $\left(\overline{g_{r}}, \phi, \xi, \eta\right)$ and the standard contact metric structure $\left({\overline{\left(g^{*}\right)}}_{1}, \phi^{*}, \xi^{*}, \eta^{*}\right)$ on $T_{r}(M, g)$.

\section{References}

[1] M. T. K. Abbassi and O. Kowalski, On g-natural metrics with constant scalar curvature on unit tangent sphere bundles, Topics in almost Hermitian geometry and related fields, 1-29, World Sci. Publ., Hackensack, NJ, 2005.

[2] E. Boeckx, When are the tangent sphere bundles of a Riemannian manifold reducible?, Trans. Amer. Math. Soc. 355 (2003), no. 7, 2885-2903.

[3] E. Boeckx and L. Vanhecke, Unit tangent sphere bundles with constant scalar curvature, Czechoslovak Math. J. 51(126) (2001), no. 3, 523-544. 
[4] Y. D. Chai, S. H. Chun, J. H. Park, and K. Sekigawa, Remarks on $\eta$-Einstein unit tangent bundles, Monatsh. Math. 155 (2008), no. 1, 31-42.

[5] J. Cheeger and D. Gromoll, On the structure of complete manifolds of nonnegative curvature, Ann. of Math. (2) 96 (1972), 413-443.

[6] J. T. Cho and J.-I. Inoguchi, Pseudo-symmetric contact 3-manifolds. II. When is the tangent sphere bundle over a surface pseudo-symmetric?, Note Mat. 27 (2007), no. 1, 119-129.

[7] S. H. Chun, J. H. Park, and K. Sekigawa, Remarks on $\eta$-Einstein tangent sphere bundles of radius $r$, preprint.

[8] O. Kowalski and M. Sekizawa, On tangent sphere bundles with small or large constant radius, Special issue in memory of Alfred Gray (1939-1998). Ann. Global Anal. Geom. 18 (2000), no. 3-4, 207-219.

[9] - On the scalar curvature of tangent sphere bundles with arbitrary constant radius, Bull. Greek Math. Soc. 44 (2000), 17-30.

[10] E. Musso and F. Tricerri, Riemannian metrics on tangent bundles, Ann. Mat. Pura Appl. (4) 150 (1988), 1-19.

[11] M. Sekizawa, Curvatures of tangent bundles with Cheeger-Gromoll metric, Tokyo J. Math. 14 (1991), no. 2, 407-417.

JEONGHYEONG PARK

DEPARTMENT OF MATHEMATics

SUNGKYUNKWAN UNIVERSITY

SUWON 440-746, KOREA

E-mail address: parkj@skku.edu

Kouei SeKigawa

Department of Mathematics

FACUlty OF SCIENCE

Nitgata University

NiIGATA, 950-2181, JAPAN

E-mail address: sekigawa@math.sc.niigata-u.ac.jp 